\title{
Decision Making Process on Multi-Objective Optimization Results
}

\author{
Marian N. Velea and Simona Lache
}

\begin{abstract}
The interpretation of results in the case of a multi-objective optimization study is partially made with the help of the trade-off curves which indicate the set of solutions that gives the best compromise between objectives. However, there is a further need for choosing one single solution from this set. This article is discussing a decision making process for selecting the most beneficial design solution with respect to a set of proportion factors applied on the objectives and defined by the end-decision makers. The results show that better trade-offs may be obtained when solving the multi-objective problem using dedicated algorithms combined with the proposed selection method instead of reducing the problem to a single-objective definition by considering weight factors.
\end{abstract}

Index Terms-Multi-objective optimization, Pareto set, negotiated design solutions.

\section{INTRODUCTION}

In the design process, the identification of the optimal solution meeting the set of requirements is of utmost importance. Although single-objective optimization problems are nowadays relatively simply solved through dedicated methods, challenges still exist when more conflicting objectives are considered. For example, one of the engineering tasks where optimization is successfully used is weight reduction, since it represents one important measure to take in order to improve the engineering systems' performance. However, weight reduction usually implies the reduction of other performance criteria such as the stiffness and strength properties or the material cost. Therefore, there are cases where many objectives need to be defined and considered within the optimization procedure in which case a conflict situation appears between objectives; this is when an increased performance in one objective leads to a decreased performance for the others [1].

Several complex techniques and algorithms have been proposed for solving such multi-objective optimization problems [2]-[5]. The weighted sum approach has been used as an attempt to simplify the problem complexity of finding solutions within multi-objective optimization problems, where all the objectives functions are summed into a single objective function, giving weight penalties for each of them [4]. Then, a solution may be obtained by running one of the many existing single-objective optimization algorithms. The main drawback regarding the weighted sum method is

Manuscript received January 27, 2015; revised July 24, 2015. This work was partially supported by the strategic grant POSDRU/159/1.5/S/137070 (2014) of the Ministry of Labor, Family and Social Protection, Romania, co-financed by the European Social Fund - Investing in People, within the Sectorial Operational Program Human Resources Development 2007-2013.

The authors are with the Transilvania University of Brasov, Brasov, Romania (e-mail: marian.velea@unitbv.ro, slache@unitbv.ro). represented by the quantification of weight penalties because the results are strongly dependent on them [6].

In order to obtain a large spectrum of solutions, dedicated multi-objective optimization algorithms are of interest [7]-[9]. One of the most spread algorithms within the current available commercial FE packages is the so-called MOGA (Multi-objective Optimization Genetic Algorithm). GRSM (Global Response Surface Method) algorithm is also used for solving multi-objective optimization problems recommended for large and time consuming models [10]. Instead of providing one single solution, the dedicated multi-objective optimization algorithms produce a set of solutions by searching within the design space for a set of Pareto optimal solutions [11]. The interpretation of results in the case of a multi-objective optimization study is partially made with the help of the trade-off curves, the so-called Pareto frontiers. The obtained Pareto frontiers only indicate the set of solutions that gives the best compromise between objectives, but there is a further need for choosing one single solution from the set. This can be done currently either by intuition or by reformulating the objectives as constraints, except one of them, or by using a composite objective function [1].

In order to deal with the difficulties of interpreting the multi-objective optimization results, this article is discussing a method in order to select the most beneficial design solutions with respect to a set of proportion factors applied on the objectives and defined by the end-decision makers, after running the optimization.

\section{METHOD}

In order to demonstrate the proposed method, let us suppose a simple multi-objective optimization problem where functions $f 1$ and $f 2$ are to be minimized, Eq. (1) and (2), and function $f 3$ is to be maximized, Eq. (3). All these functions take values in terms of $x \in[1,100]$ which represents the design variable.

$$
\begin{gathered}
f_{1}=\frac{x^{2}}{200} . \\
f_{2}=\frac{100}{\sqrt{x}} . \\
f_{3}=20 \log x .
\end{gathered}
$$

The above considered functions are graphically illustrated within Fig. 1.

The goal is to find the value of the design variable $x$ that may offer the best trade-off solution between the conflicting objective functions in terms of proportion factors defined by 
decision makers. The resulted Pareto frontiers are shown within Fig. 2 and Fig. 3. The trade-off between two objectives may be realized between the points that define the Pareto optimal set, in terms of the objective importance. However, when it comes to multiple objectives, difficulties with using the Pareto front arise from the fact that the best compromise between two objectives does not necessarily represent the best one between other two objectives. Therefore, an overall performance of the objectives is needed, that relates the contribution of each objective when searching the most beneficial overall solution.

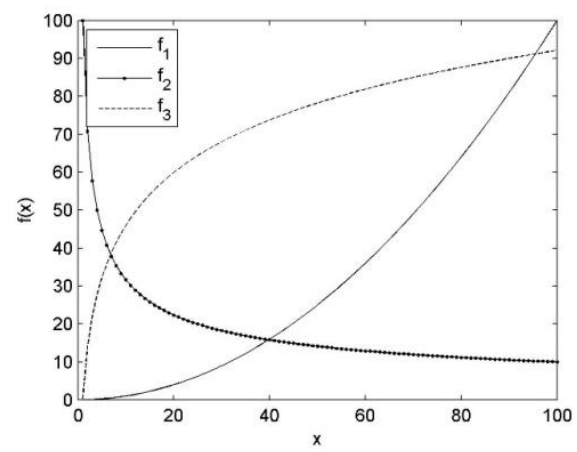

Fig. 1. Considered functions within the defined multi-objective optimisation problem.

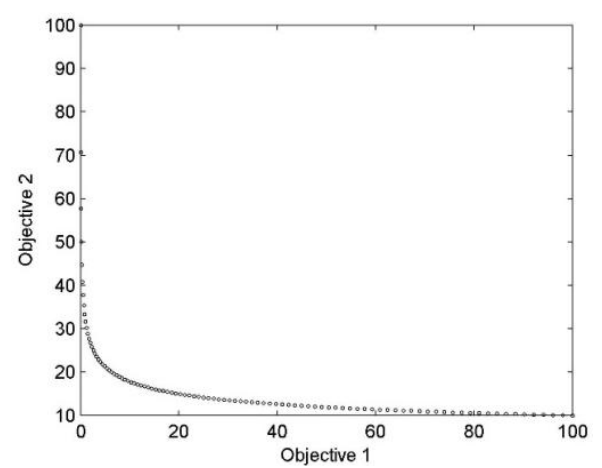

Fig. 2. Pareto front-trade-off solutions between Obj. 1 and Obj. 2.

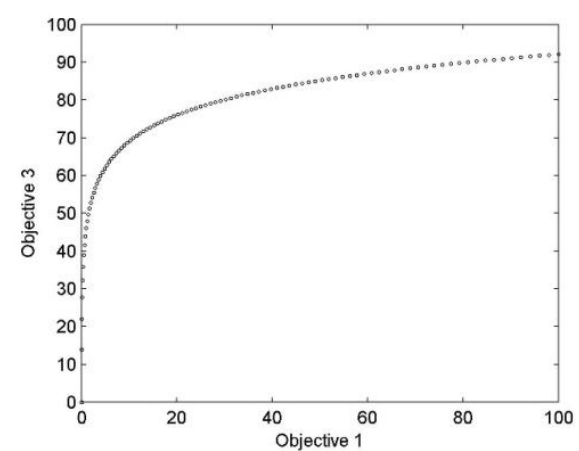

Fig. 3. Pareto front-trade-off solutions between Obj. 1 and Obj. 3.

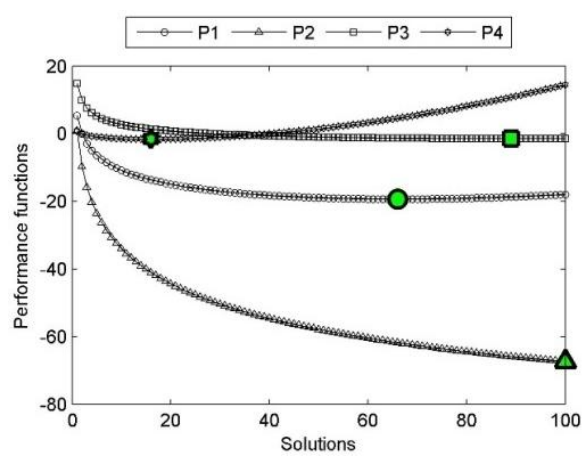

Fig. 4. Overall performance functions and their corresponding minimum values.
Such an overall performance function $P$ is adapted here from [1], where proportion factors are applied to relate the contribution of the objectives, Eq. (4):

$$
P=\frac{\sum_{i=1}^{n} p_{i} \times \xi_{i}-\sum_{j=1}^{m} p_{j} \times \xi_{j}}{100}
$$

where:

$p_{i}$ and $p_{j}$ represents the proportions to which the value of the $i^{\text {th }}$ objective (to be minimized) and the value of the $j^{\text {th }}$ objective (to be maximized) contributes to the overall performance function $P$, in percent;

$$
\begin{gathered}
-\sum_{i=1}^{n} p_{i}+\sum_{j=1}^{m} p_{j}=100 \% . \\
-\xi_{i}=\frac{\left(\overline{o_{i}}-o_{i_{\min }}\right)}{o_{i_{\max }}-o_{i_{\min }}}, \xi_{i} \in[0,1] ; \quad \xi_{j}=\frac{\left(\overline{o_{j}}-o_{j_{\min }}\right)}{{o_{j_{\max }}-o_{j_{\min }}}_{\xi_{j}}},
\end{gathered}
$$

$\bar{O}_{i}=\frac{O_{i}}{O_{i_{r e f}}}$, represents the normalized value of the $i^{\text {th }}$ objective to the reference value, $i=1, \ldots, n$;

$\bar{O}_{j}=\frac{o_{j}}{o_{j_{r e f}}}$, represents the normalized value of the $j^{\text {th }}$ objective to the reference value, $j=1, \ldots, m$;

The objective's reference value is calculated by assuming an initial value for the design variable $x_{\text {init }}: O_{i_{\text {ref }}}=f_{i}\left(x_{\text {init }}\right)$; $o_{j_{\text {ref }}}=f_{j}\left(x_{\text {init }}\right)$

$$
O_{i}=f_{i}(x) ; O_{j}=f_{j}(x)
$$

$O_{i_{\min }}, O_{i_{\max }}-$ minimum and maximum values of the objective to be minimized, $i=1, \ldots, n$;

$O_{j_{\min }}, O_{j_{\max }}$-minimum and maximum values of the objective to be minimized, $j=1, \ldots, m$;

Fig. 4 shows the resulted overall performance functions $P$ by considering 4 cases with different distributions of the proportion factors pi and $p j$, Table I, and an initial value of 45 for $x$. The minimum value of the overall performance functions $P$ gives the best compromise between the considered objectives while taking into account the desired value for the proportions pi and $p j$.

The obtained values for the objective functions are graphically presented within Fig. 5 and Fig. 6. For the objectives to be minimized, improvements are observed if their normalized value is below 1 , while a value above 1 indicates improvements of the objectives to be maximized. Table I, correlated with Fig. 5 and Fig. 6, shows a selection of four possible solutions obtained by varying the proportion factors $p_{i}$ and $p_{j}$ in such a way to give different contributions of the objective functions to the obtained design solution.

The plots shown, Fig. 5 and Fig. 6, are divided into four regions (I - IV) in order to clearly show and classify the performance offered by each of the design solutions. The performance is increased for both of the objectives if the solution comes from region I or it is decreased for both of the objectives if the solution comes from region IV. Region II and III contain those solutions where only one of the objectives has an increased performance.

The solution denoted $S 1$ shows a tied trade-off between 
objectives, all having equal influence on the solution $(33 \%$ each), Table I. $S 2-S 4$ represent extreme dominated solutions: $S 2$ represents Objective 3 dominated solution $(p=90 \%)$. S3 represent Objective 2 dominated solution $(p=90 \%)$. $S 4$ represent Objective 1 dominated solution $(p=90 \%)$, Table I.
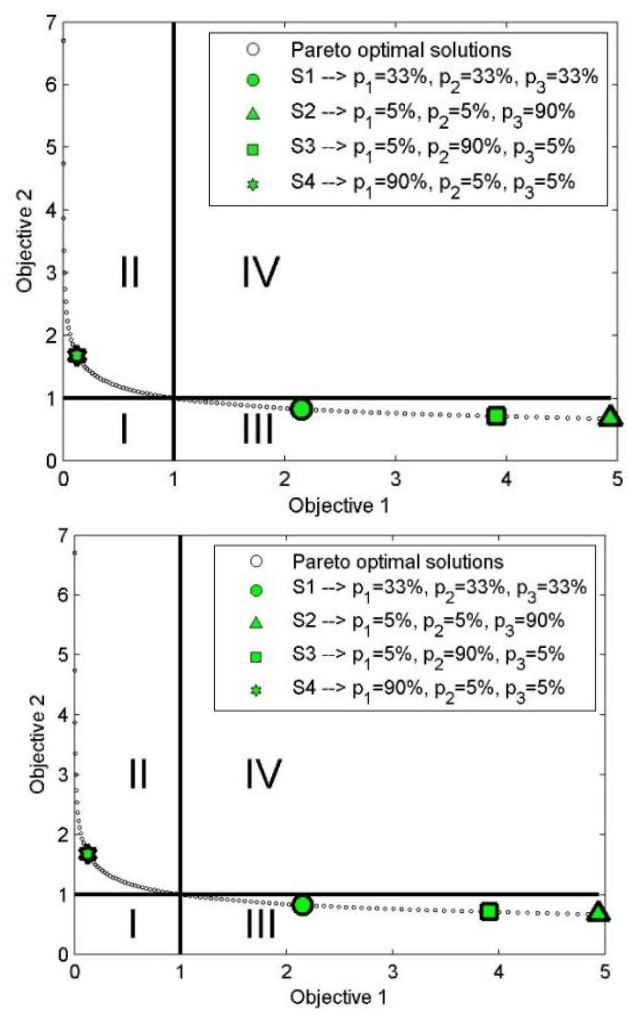

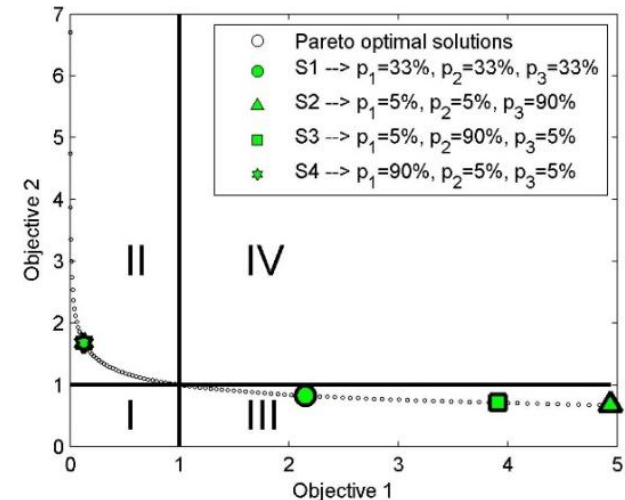

Fig. 5. Obj. 1 vs. Obj. 2 - Selected solutions using the herein proposed method.

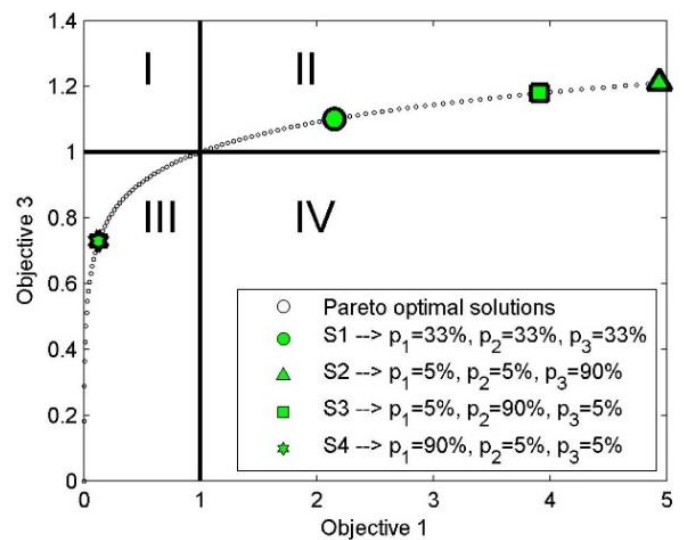

Fig. 6. Obj. 1 vs. Obj. 3 - Selected solutions using the herein proposed method.

TABLE I: RESPONSES’ VALUES AND THEIR CORRESPONDING PROPORTION FACTORS FOR DIFFERENT DESIGN SOLUTIONS

\begin{tabular}{|c|c|c|c|c|c|c|c|c|}
\hline \multirow{3}{*}{ Responses } & \multicolumn{8}{|c|}{ Solutions } \\
\hline & \multicolumn{2}{|c|}{ S1 } & \multicolumn{2}{|c|}{ S2 } & \multicolumn{2}{|c|}{ S3 } & \multicolumn{2}{|c|}{ S4 } \\
\hline & Obj. value & $p \%$ & Obj. value & $p \%$ & Obj. value & $p \%$ & Obj. value & $p \%$ \\
\hline$f_{1}(x)$ & 2.15 & 33 & 4.94 & 5 & 3.91 & 5 & 0.13 & 90 \\
\hline$f_{2}(x)$ & 0.83 & 33 & 0.67 & 5 & 0.71 & 90 & 1.7 & 5 \\
\hline$f_{3}(x)$ & 1.1 & 33 & 1.21 & 90 & 1.18 & 5 & 0.73 & 5 \\
\hline$P_{s}$ & \multicolumn{2}{|c|}{-19.31} & \multicolumn{2}{|c|}{-67.43} & \multicolumn{2}{|c|}{-1.43} & \multicolumn{2}{|c|}{-1.62} \\
\hline$x$ & \multicolumn{2}{|c|}{66} & \multicolumn{2}{|c|}{100} & \multicolumn{2}{|c|}{89} & \multicolumn{2}{|c|}{16} \\
\hline
\end{tabular}

\section{CASE STUDY}

The above presented method is applied further on within a simple case study. The problem consists in minimizing 3 conflicting objectives related to a cantilever beam, Fig. 7: min: $m$ - mass of the beam; $\min \delta_{z}$-deflection at the free-end when applying the force $F_{z}$; $\min \varphi_{x}$-rotation at the free-end when applying the torque $T$. The optimization model was defined within Excel and it was solved using MOGA within the Hyperstudy® facilities.

The optimization results may be interpreted from Fig. 8 and Fig. 9, correlated with Table II. The solution denoted S1 represents a tied negotiation between the three considered objectives (the contribution of all the objective functions is $33 \%)$. $S 2$ represents rotation $\varphi_{x}$ dominated solution $(p=90 \%)$. $S 3$ represent displacement $\delta_{z}$ dominated solution $(p=90 \%)$. $S 4$ represent the mass dominated solution $(p=90 \%)$.

The same optimization model as previously described was further on solved by using the ARSM algorithm following the weighted sum approach. The results are represented by single values given to the model responses shown within Table III. By comparing Table III with Table II, it is concluded that better trade-offs may be obtained when solving the multi-objective problem using dedicated algorithms combined with the proposed selection method instead of reducing the problem to a single-objective definition by considering weight factors.

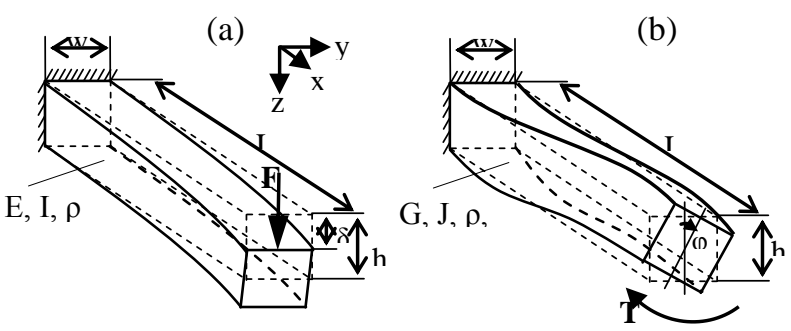

Fig. 7. Bending of a cantilever beam (a) and Torsion of cantilever beam (b) $w$ and $h$ are design variables, while $\delta_{z}, \varphi_{x}$ and the $m$ (mass of the beam) represent the responses considered as objectives to be minimized. 
TABLE II: RESPONSES' VALUES AND THEIR CORRESPONDING PROPORTION FACTORS FOR DiFFERENT DeSIGN SOLUTIONS OBTAINED By USING THE PROPOSED SELECTION METHOD

\begin{tabular}{|c|c|c|c|c|c|c|c|c|}
\hline \multirow{3}{*}{ Responses } & \multicolumn{8}{|c|}{ Solutions } \\
\hline & \multicolumn{2}{|c|}{ S1 } & \multicolumn{2}{|c|}{ S2 } & \multicolumn{2}{|c|}{ S3 } & \multicolumn{2}{|c|}{ S4 } \\
\hline & Obj. value & $p \%$ & Obj. value & $p \%$ & Obj. value & $p \%$ & Obj. value & $p \%$ \\
\hline mass [-] & 1.07 & 33 & 1.49 & 5 & 1.48 & 5 & 0.91 & 90 \\
\hline$\delta_{z}[-]$ & 0.66 & 33 & 0.92 & 5 & 0.18 & 90 & 1.24 & 5 \\
\hline$\varphi_{x}[-]$ & 1.16 & 33 & 0.22 & 90 & 1.17 & 5 & 1.15 & 5 \\
\hline$P_{s}[-]$ & \multicolumn{2}{|c|}{5.52} & \multicolumn{2}{|c|}{1.21} & \multicolumn{2}{|c|}{1.58} & \multicolumn{2}{|c|}{10.44} \\
\hline$w[\mathrm{~m}]$ & \multicolumn{2}{|c|}{0.045} & \multicolumn{2}{|c|}{0.087} & \multicolumn{2}{|c|}{0.038} & \multicolumn{2}{|c|}{0.049} \\
\hline$h[\mathrm{~m}]$ & \multicolumn{2}{|c|}{0.059} & \multicolumn{2}{|c|}{0.043} & \multicolumn{2}{|c|}{0.098} & \multicolumn{2}{|c|}{0.047} \\
\hline
\end{tabular}

TABLE III: RESPONSES' VALUES OBTAINED By USING THE WEIGTHED SUM APPROACH

\begin{tabular}{|c|c|c|c|c|c|c|c|c|}
\hline \multirow{3}{*}{ Responses } & \multicolumn{8}{|c|}{ Solutions } \\
\hline & \multicolumn{2}{|c|}{ S1 } & \multicolumn{2}{|c|}{ S2 } & \multicolumn{2}{|c|}{ S3 } & \multicolumn{2}{|c|}{ S4 } \\
\hline & Obj. value & weight & Obj. value & weight & Obj. value & weight & Obj. value & weight \\
\hline mass [-] & 0.84 & 0.33 & 0.96 & 0.05 & 1.44 & 0.05 & 0.84 & 0.9 \\
\hline$\delta_{z}[-]$ & 1.64 & 0.33 & 1.32 & 0.05 & 0.19 & 0.9 & 1.64 & 0.05 \\
\hline$\varphi_{x}[-]$ & 1.19 & 0.33 & 0.89 & 0.9 & 1.2 & 0.05 & 1.2 & 0.05 \\
\hline$w[\mathrm{~m}]$ & \multicolumn{2}{|c|}{0.049} & \multicolumn{2}{|c|}{0.054} & \multicolumn{2}{|c|}{0.038} & \multicolumn{2}{|c|}{0.049} \\
\hline$h[\mathrm{~m}]$ & \multicolumn{2}{|c|}{0.042} & \multicolumn{2}{|c|}{0.044} & \multicolumn{2}{|c|}{0.095} & \multicolumn{2}{|c|}{0.042} \\
\hline
\end{tabular}
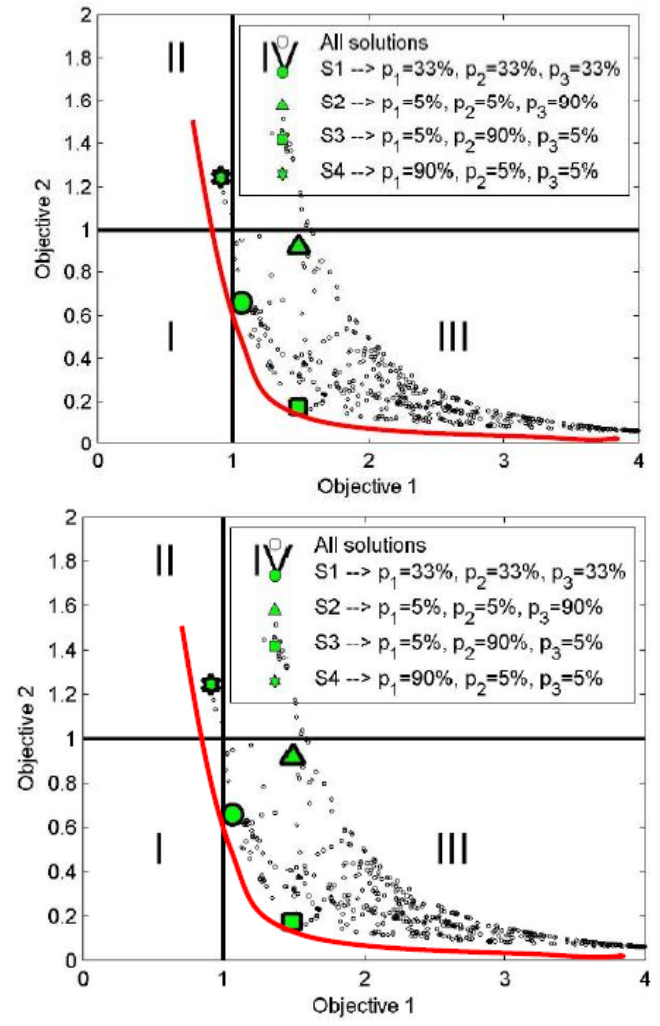

Fig. 8. Obj.1 - mass vs. Obj. $2-\delta_{z} ;$ Selected solutions using the proposed method.

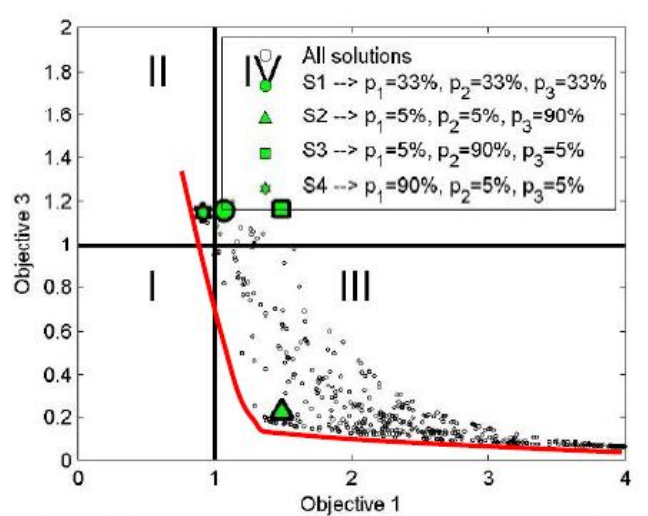

Fig. 9. Obj.1 - mass vs. Obj. $3-\varphi_{x}$; Selected solutions using the proposed method.

\section{CONCLUSION}

The proposed selection method allows searching within Pareto optimal sets for the most beneficial solutions by following the importance allocated for each of the objectives, using the so-called proportion factors. The procedure is therefore following a multi-objective optimisation solved by using dedicated multi-objective algorithms such as MOGA or GRSM algorithms that generates Pareto optimal solutions. Compared to the weighted sum approach, the dedicated multi-objective algorithms combined with the proposed selection method will take longer time to reach the solution. However, the main advantage of using the proposed method comes from the fact that it allows exploring the full Pareto optimal design solutions which gives the complete trade-off information for the end-decision makers and possibly the chance to select a better compromise.

\section{ACKNOWLEDGMENT}

This work was partially supported by the strategic grant POSDRU/159/1.5/S/137070 (2014) of the Ministry of Labor, Family and Social Protection, Romania, co-financed by the European Social Fund - Investing in People, within the Sectorial Operational Program Human Resources Development 2007-2013.

\section{REFERENCES}

[1] M. F. Ashby, "Multi-objective optimization in material design and selection," Acta Materialia, vol. 48, pp. 359-369, 2000.

[2] R. T. Marler and J. S. Arora, "Survey of multi-objective optimization methods for engineering. Structural and multidisciplinary optimization," Structural and Multidisciplinary Optimization, vol. 26, pp. 369-395, 2004.

[3] P. Badalló, D. Trias, L. Marín, and J. A. Mayugo, "A comparative study of genetic algorithms for the multi-objective optimization of composite stringers under compression loads," Composites Part B: Engineering, vol. 47, pp. 130-136, 2013.

[4] M. Walker and R. E. Smith, "A technique for the multiobjective optimisation of laminated composite structures using genetic algorithms and finite element analysis," Composite Structures, vol. 62, pp. 123-128, 2003.

[5] K. Wang, D. Kelly, and S. Dutton, "Multi-objective optimisation of composite aerospace structures," Composite Structures, vol. 57, pp. 141-148, 2002. 
[6] M. Kaufmann, D. Zenkert, and P. Wennhage, "Integrated cost/weight optimization of aircraft structures," Structural and Multidisciplinary Optimization, vol. 41, pp. 325-334, 2010.

[7] D. S. Lee, C. Morillo, G. Bugeda, S. Oller, and E. Onate, "Multilayered composite structure design optimisation using distributed/parallel multi-objective evolutionary algorithms," Composite Structures, vol. 94, pp. 1087-1096, 2012.

[8] F. S. Almeida and A. M. Awruch, "Design optimization of composite laminated structures using genetic algorithms and finite element analysis," Composite Structures, vol. 88, pp. 443-454, 2009.

[9] J. Pajot, "Optimal design exploration using global response surface method," Altair, 2013.

[10] Altair, Help and Documentation Hyperworks, December 2014.

[11] A. Konak, D. W. Coit, and A. E. Smith, "Multi-objective optimization using genetic algorithms: A tutorial," Reliability Engineering \& System Safety, vol. 9, pp. 992-1007, 2006.

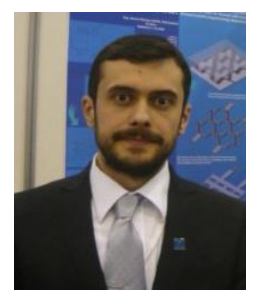

Marian N. Velea received his $\mathrm{PhD}$ in mechanical engineering from Transilvania University of Brașov, Romania in 2011. From 2012-2013 he worked as a post-doc researcher within the lightweight structures research group from the Aeronautical and Vehicle Engineering Department of KTH, Royal Institute of Technology, Sweden. He is currently an assistant professor at Transilvania University of Brașov, Romania.

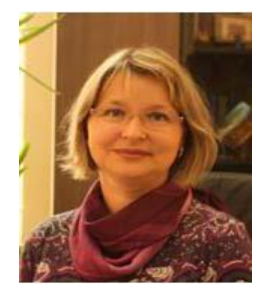

Simona Lache received her $\mathrm{PhD}$ in mechanical engineering from Transilvania University of Brașov, Romania in 2000. She has been an academic staff at UTBv since graduation, as a full professor since 2006 and the $\mathrm{PhD}$ supervisor since 2007. She holds the position of vice-rector since 2008 , being responsible for internationalization and quality assurance. 